\section{Langzeitüberleben nach venovenöser ECMO}

Enger TB et al. Long-term survival in adult patients with severe acute lung failure receiving veno-venous extracorporeal membrane oxygenation. Crit Care Med 2017; 45: 1718 - 1725

Eine venovenöse ECMO (extrakorporale Membranoxygenierung) kann bei schwerer respiratorischer Insuffizienz nach Versagen konventioneller Maßnahmen lebensrettend sein. Die Sterblichkeit der Patienten in der Klinik bleibt aber hoch und liegt je nach Veröffentlichung bei bis zu $62 \%$.

In Anbetracht des immer häufigeren Einsatzes der ECMO - zwischen 2007 und 2012 hat er sich etwa verdreifacht wird aber auch die Langzeitprognose der Patienten nach Entlassung aus der Klinik immer wichtiger. Bislang gibt es dazu nur wenige Untersuchungen; Mediziner aus Regensburg legen jetzt Zahlen vor.

Tone Bull Enger und ihre Kollegen haben Daten von insgesamt 553 Patienten herangezogen, bei denen zwischen 2007 und 2016 an der Universitätsklinik Regensburg eine venovenöse ECMO erfolgt war. Davon waren 336 Patienten (60,8\%) nach erfolgreicher Behandlung aus dem Krankenhaus entlassen worden, $217 \mathrm{~Pa}$ tienten waren in der Klinik gestorben.

Bei ihnen prüften sie, wie viele Patienten im Januar 2017 noch am Leben waren und verglichen die Sterblichkeit mit der nach Alter und Geschlecht gematchten deutschen Allgemeinbevölkerung. Darüber hinaus befragten sie diese ehemaligen Patienten nach ihrer funktionellen Unabhängigkeit im Alltag anhand des
Leistungsstatus der Eastern Cooperative Oncology Group (ECOG); er reicht von 0 (keine Einschränkungen) bis 5 (Tod). Schließlich suchten die Wissenschaftler nach Faktoren, die unabhängig mit dem Langzeitüberleben verbunden waren.

Die Auswertung ergab nach einer medianen Nachbeobachtungszeit von knapp 5 Jahren weitere 69 Todesfälle (12,5\%). Im Zeitraum 2007 - 2013 (spätere Daten waren nicht verfügbar) starben $36 \mathrm{~Pa}$ tienten, das waren fast 10-mal so viel wie in der vergleichbaren Allgemeinbevölkerung (standardisierte Sterberate 9,4).

Für die Gesamtkohorte errechneten sich in den Kaplan-Meier-Schätzungen Überlebensraten von

- $62 \%$ nach 30 Tagen,

- $57 \%$ nach 90 Tagen

- $52 \%$ nach 1 Jahr und

- $46 \%$ nach 5 Jahren.

Wurden nur diejenigen Patienten berücksichtigt, die aus der Klinik entlassen worden waren, lagen die Werte dagegen bei
- $99 \%$ nach 30 Tagen
- $95 \%$ nach 90 Tagen,
- $86 \%$ nach 1 Jahr und
- $76 \%$ nach 5 Jahren.

Zum Ende der Nachbeobachtungszeit beschrieben 185 Patienten (69\%) einen ECOG-Status von 0 , weiter $21 \%$ einen Status von 1, entsprechend einer funktionellen Einschränkung nur bei körperlicher Anstrengung.

In der multivariaten Analyse errechnete sich für die Gesamtkohorte höheres Alter, eingeschränkte immunologische Funktion und Schwere der Erkrankung als mit dem Langzeitüberleben verbunden. Beschränkte man die Auswertung jedoch auf die 336 Patienten, die lebend aus der Klinik entlassen worden waren, blieben nur noch Alter, Störung des Immunsystems - solide und hämatologische Malignome, Behandlung mit Immunsuppressiva und HIV-Infektion und funktionelles Ergebnis bei Entlassung anhand der Glasgow Coma Scale als unabhängig mit der Sterblichkeit assoziiert. Tendenziell, aber knapp nicht signifikant zeigten Patienten nach Traumata bessere Langzeitüberlebensraten als Patienten mit anderen Erkrankungen.

FAZIT

Wenn Patienten eine venovenöse ECMO - bzw. die zugrunde liegende Erkrankung - bis zur Entlassung aus der Klinik überleben, sind die weiteren Überlebenschancen relativ gut, so die Autoren. Und dieses Überleben wird nicht mit einer hohen Rate funktioneller Abhängigkeit erkauft - bei $90 \%$ der Patienten bestanden langfristig keine oder allenfalls geringfügige Einschränkungen im Alltag. Diese Zahlen könnten den behandelnden Ärzten helfen, Patienten und ihre Angehörigen adäquat aufzuklären.

Dr. Elke Ruchalla, Bad Dürrheim 